\title{
Evaluation of the Larvicidal Efficacy of Five Indigenous Weeds against an Indian Strain of Dengue Vector, Aedes aegypti L. (Diptera: Culicidae)
}

\author{
Aarti Sharma, ${ }^{1}$ Sarita Kumar, ${ }^{2}$ and Pushplata Tripathi ${ }^{1}$ \\ ${ }^{1}$ School of Life Sciences, Indira Gandhi National Open University, Maidan Garhi, New Delhi 110068, India \\ ${ }^{2}$ Department of Zoology, Acharya Narendra Dev College, University of Delhi, Kalka Ji, New Delhi 110019, India \\ Correspondence should be addressed to Sarita Kumar; saritakumar@andc.du.ac.in
}

Received 30 September 2015; Accepted 11 January 2016

Academic Editor: Bernard Marchand

Copyright ( $\odot 2016$ Aarti Sharma et al. This is an open access article distributed under the Creative Commons Attribution License, which permits unrestricted use, distribution, and reproduction in any medium, provided the original work is properly cited.

Background and Objectives. Aedes aegypti, dengue fever mosquito, is primarily associated with the transmission of dengue and chikungunya in tropical and subtropical regions of the world. The present investigations were carried out to assess the larvicidal efficiency of five indigenous weeds against Ae. aegypti. Methods. The 1,000 ppm hexane and ethanol extracts prepared from the leaves and stem of five plants (Achyranthes aspera, Cassia occidentalis, Catharanthus roseus, Lantana camara, and Xanthium strumarium) were screened for their larvicidal activity against early fourth instars of dengue vector. The extracts which could cause $80-100 \%$ mortality were further investigated for their efficacy. Results. The preliminary screening established the efficacy of hexane extracts as compared to the ethanol extracts. Further investigations revealed the highest larvicidal potential of $A$. aspera extracts exhibiting $\mathrm{LC}_{50}$ value of $82.555 \mathrm{ppm}$ and $68.133 \mathrm{ppm}$, respectively. Further, their leaf extracts showed 5-85.9\% higher larvicidal activity and stem extracts exhibited 0.23 - to 0.85 -fold more efficiency than the other four extracts. Conclusion. The present investigations suggest the possible use of A. aspera as an ideal ecofriendly, larvicidal agent for the control of dengue vector, Ae. aegypti. Future studies are, however, required to explore and identify the bioactive component involved and its mode of action.

\section{Introduction}

Mosquitoes have attracted considerable attention worldwide being the most prevalent vectors of several lethal diseases, malaria, filariasis, chikungunya, yellow fever, dengue, and encephalitis, accounting for enormous mortality and morbidity. Since last few years, dengue fever has become the major public-health concern in tropical and subtropical regions of the world. It is considered the most rapidly spreading mosquito-borne disease with 30 -fold rise in global occurrence since the past 5 decades. The incidence of dengue infections estimated by World Health Organization is about 390 million annually of which 96 million are supposed to be manifested clinically [1]. As per WHO reports, approximately 3900 million individuals, inhabiting over 128 endemic countries, are likely to be at the risk of dengue. In India, official records of the Union Health Ministry reveal a massive increase in dengue infections every year [2].
Till date, specific medications and vaccinations are not available commercially for treating dengue fever. The only approach followed to reduce the incidence of dengue is by the control of its vector, Aedes aegypti L., which is also the primary carrier of chikungunya virus and yellow fever virus. In the past, the control measures for mosquito vectors were based on the frequent and indiscriminate use of synthetic chemical-based insecticides, such as organochlorines, carbamates, organophosphates, and pyrethroids [3]. Nevertheless, the blind use of insecticides has resulted in the increased selection pressure on the mosquitoes leading to the development of insecticide resistance in them $[4,5]$. Varying amount of resistance to commonly used insecticides, temephos, fenthion, malathion, and dichlorodiphenyltrichloroethane (DDT), has been reported by Tikar et al. [6]. In addition, it has raised many other concerns including toxicity to human beings, harm to nontarget population, long persistence in environment, and entry in the food chain [7]. 
Keeping in view the increasing documentation of negative environmental and health impact of synthetic insecticides and increasingly rigorous environmental directives about use of pesticides, the researchers have transformed their interest towards the development and use of botanical pest management products for controlling mosquitoes and other insects [8]. Botanicals are considered safe alternative to synthetic pesticides since they are biodegradable and safe for environment causing low toxicity to humans and nontarget organisms [9]. More than 2,000 plants species have already been known to possess chemical factors and metabolites of significance in pest control programs whilst products of approximate 344 species have been reported to encompass diverse activities against mosquitoes $[10,11]$. A number of such plant products have been used for insect control since primordial time. Biologically active plant extracts have been well recognized for formulating an ecologically sound and environmentally accepted mosquito control program; more studies are being carried out to identify variety of bioeffective substances found in different plant species [8]. Several reports have established the efficacy of plant extracts and essential oils as efficient mosquito larvicides and repellents, without posing hazards of toxicity to humans [12-15]. Nevertheless, the mosquito control potential of weed plants has not been attempted at large scale leading to the availability of limited reports concerning the usefulness and promising use of certain weeds against Ae. aegypti as larvicidal, ovicidal, and oviposition deterrent agents [5, 16-18].

Weeds are nowadays preferable plants of research because of their easy availability, cultivability, undesirability, and need of management. Moreover, the beneficial utilization of weeds as mosquito control agents can boost the weed management programs. The present study assesses the mosquito control potential of five common indigenous weeds, Achyranthes aspera, Cassia occidentalis, Catharanthus roseus, Lantana camara, and Xanthium strumarium. Achyranthes aspera (Amaranthaceae), commonly known as prickly chaff flower, is an important medicinal herb found commonly on the roadsides throughout India. The extracts prepared from the leaves, flowers, and the seeds of $A$. aspera have been reported to possess biological activity against the fourth instars of Anopheles subpictus and Culex tritaeniorhynchus [19]. Certain bioactive components derived from this plant have been found to exhibit larvicidal, pupicidal, and growth regulatory activity against insects [20]. Cassia occidentalis (Fabaceae) is an erect, lightly branched leguminous tree/shrub, commonly known as Coffee Senna or Coffee Weed, which is found in tropical, subtropical, and semiarid regions. In India it is widely prevalent as an opportunist that grows abundantly along roadsides immediately after rains [21]. The leaf extracts of $C$. occidentalis have been found to have effective activity against Anopheles sp. and Cx. quinquefasciatus larvae [22, 23]. Likewise, Catharanthus roseus (L.) (fam., Apocynaceae; formerly Vinca rosea L.), naturalised in all tropical countries and invasive in parts of Kenya, is reported to exhibit excellent control potential against Ae. aegypti, An. stephensi, and Cx. quinquefasciatus $[9,24]$. Another weed selected for the current study, Lantana camara (Verbenaceae), commonly known as Wild Sage, an aggressive, obligate breeder weed that has invaded vast areas in many tropical and subtropical regions, has been regarded as one of the 10 most noxious weeds in the world [25]. L. camara has also been reported to possess mosquito larvicidal and adulticidal activity against Ae. aegypti, Cx. quinquefasciatus, An. culicifacies, An. fluviatilis, and An. stephensi [26]. On the other hand, Xanthium strumarium (fam., Asteraceae; chotadhatura), which grows in tropical environments and is particularly abundant in India, Pakistan, and Sri Lanka, offers larvicidal and repellent potential against An. culicifacies Giles species A, An. Stephensi, Cx. quinquefasciatus, and Ae. aegypti [27].

Keeping in view above properties and possible mosquito control potential of these weeds, the leaves and stems of all these five weeds were assessed for their larvicidal potential against an Indian strain of dengue vector, Ae. aegypti. The investigations were carried out with an objective that the result of the study could be useful in promoting research aiming at the development of new eco-friendly alternative for mosquito control based on the biologically active plant sources.

\section{Materials and Methods}

2.1. Rearing of Mosquitoes. The present investigations employed the larvae of dengue fever mosquito, Ae. aegypti, which were collected from fields of Delhi and surrounding areas. Rearing of the mosquito vector was carried out in a laboratory maintained at $28 \pm 1^{\circ} \mathrm{C}, 80 \pm 5 \% \mathrm{RH}$ and $14: 10 \mathrm{~L} / \mathrm{D}$ photoperiod as per the protocol described by Kumar et al. [28].

2.2. Collection of Plant Material. Five plant species of weeds were identified for the present investigations keeping in mind collecting species which were neither threatened nor endangered nor endemic. Moreover, selection of the plants was based on the literature survey, their easy availability, and uncomplicated cultivation being competent and invasive in nature. The five species of plants selected, A. aspera, C. occidentalis, C. roseus, L. camara, and X. strumarium, belonged to different families and also carried some medicinal importance.

The plants were collected from different sites of South Delhi, India (Figure 1), and brought to the laboratory in sterile polythene bags. The leaves and stems of each plant were separated and thoroughly washed with distilled water to clean dust or any other undesirable particles stuck to them. The plant parts were also carefully scrutinized for any disease or infection. The selected healthy parts were shade-dried at room temperature $\left(27 \pm 2^{\circ} \mathrm{C}\right)$ for approximate three weeks till they were dried completely. The five plant species and the parts used in the present investigations are summarized in Table 1.

2.3. Preparation of Plant Extracts. Plant extracts were prepared by maceration method in which the dried plant parts were ground with electric blender and then sieved to get fine powder. The $50 \mathrm{~g}$ of each dried and powdered part was weighed and soaked in $200 \mathrm{~mL}$ of ethanol and hexane solvent, separately in a stoppered conical flask $(500 \mathrm{~mL})$ for a definite 
TABLE 1: Screening of the $1000 \mathrm{ppm}$ hexane and ethanol extracts of five plant species for their larvicidal activity against early fourth instars of dengue vector Aedes aegypti.

\begin{tabular}{|c|c|c|c|c|c|}
\hline \multirow{2}{*}{ Name of the plant species } & \multirow{2}{*}{ Local name } & \multirow{2}{*}{ Family } & \multirow{2}{*}{ Part used } & \multicolumn{2}{|c|}{$\%$ mortality after $24 \mathrm{~h}$} \\
\hline & & & & Hexane extract & Ethanolic extract \\
\hline \multirow{2}{*}{ Achyranthes aspera } & \multirow{2}{*}{ Prickly chaff flower } & \multirow{2}{*}{ Amaranthaceae } & Leaves & $100.0 \pm 0.0^{*}$ & $11.0 \pm 0.0$ \\
\hline & & & Stems & $100.0 \pm 0.0$ & $19.0 \pm 0.33$ \\
\hline \multirow{2}{*}{ Cassia occidentalis } & \multirow{2}{*}{ Chakunda; Coffee Senna; Coffee Weed } & \multirow{2}{*}{ Fabaceae } & Leaves & $100.0 \pm 0.0$ & $16.0 \pm 0.0$ \\
\hline & & & Stems & $100.0 \pm 0.0$ & $10.0 \pm 0.0$ \\
\hline \multirow{2}{*}{ Catharanthus roseus } & \multirow{2}{*}{ Periwinkle } & \multirow{2}{*}{ Apocynaceae } & Leaves & $100.0 \pm 0.0$ & $50.0 \pm 0.0$ \\
\hline & & & Stems & $100.0 \pm 0.0$ & $05.0 \pm 0.0$ \\
\hline \multirow{2}{*}{ Lantana camara } & \multirow{2}{*}{ Spanish flag; Wild Sage; West Indian lantana } & \multirow{2}{*}{ Verbenaceae } & Leaves & $100.0 \pm 0.0$ & $11.0 \pm 0.0$ \\
\hline & & & Stems & $100.0 \pm 0.0$ & $05.0 \pm 0.0$ \\
\hline \multirow{2}{*}{ Xanthium strumarium } & \multirow{2}{*}{ Common cocklebur; chotadhatura } & \multirow{2}{*}{ Asteraceae } & Leaves & $100.0 \pm 0.0$ & $41.0 \pm 4.33$ \\
\hline & & & Stems & $100.0 \pm 0.0$ & $47.0 \pm 4.0$ \\
\hline
\end{tabular}

${ }^{*}$ Mean \pm SEM.

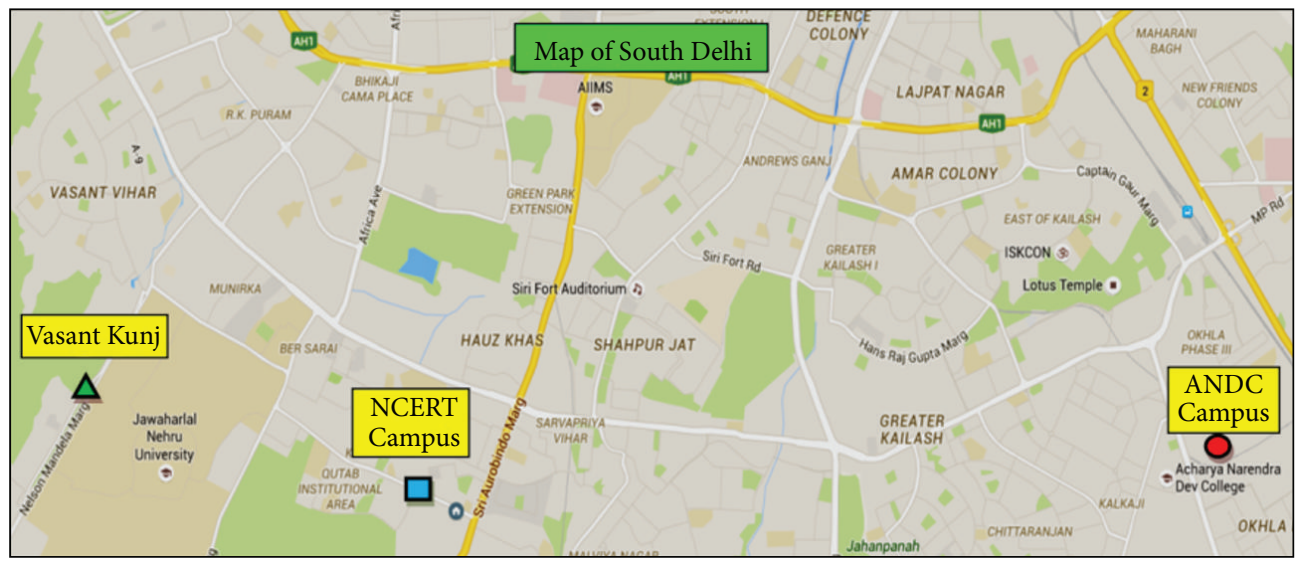

FIGURE 1: Different sites of the collection of different plant species in South Delhi, India. @ ANDC Campus [Latitude, Longitude (28.538548, $77.262919) 28^{\circ} 32^{\prime} 18.7728^{\prime \prime} \mathrm{N}$ and $77^{\circ} 15^{\prime} 46.5084^{\prime \prime} \mathrm{E}$ ] (Catharanthus roseus). - NCERT Campus [Latitude, Longitude (28.538037, 77.192734) $28^{\circ} 32^{\prime} 16.9332^{\prime \prime} \mathrm{N}$ and $77^{\circ} 11^{\prime} 33.8424^{\prime \prime} \mathrm{E}$ ] (Lantana camara). A Vasant Kunj [Latitude, Longitude $(28.547015,77.161265) 28^{\circ} 32^{\prime} 49.2540^{\prime \prime} \mathrm{N}$ and $\left.77^{\circ} 9^{\prime} 40.5540^{\prime \prime} \mathrm{E}\right]$ (Achyranthes aspera, Cassia occidentalis, and Xanthium strumarium).

period of time of 5-7 days with frequent agitation until soluble matter was dissolved. The extracts, thus obtained, were concentrated using a vacuum evaporator (Buchi Type) under low pressure keeping the temperature not more than their respective boiling point, that is, $78^{\circ} \mathrm{C}$ and $60^{\circ} \mathrm{C}$. The concentrated extracts were stored in a refrigerator at $4^{\circ} \mathrm{C}$ as the stock solution of $1000 \mathrm{ppm}$ prepared using ethanol as the solvent.

2.4. Screening of Extracts for Larvicidal Efficacy against Ae. aegypti. The larvicidal efficacy of all the extracts was investigated in two phases. In the first phase, the toxicity potential of $1000 \mathrm{ppm}$ of each extract was screened against early fourth instars of Ae. aegypti. For screening, the active early fourth instars of Ae. aegypti were separated in batches of 20. Each batch was transferred in separated bowls containing $99 \mathrm{~mL}$ of distilled water which were then transferred to glass jars containing $100 \mathrm{~mL}$ of distilled water and $1 \mathrm{~mL}$ of an extract at $1000 \mathrm{ppm}$. Each bioassay had three replicates carried out concurrently at comparable conditions. Controls were exposed to the solvent, that is, ethanol alone.

The dead and moribund larvae were recorded after an exposure period of $24 \mathrm{~h}$. The larvae were touched gently with the help of a glass rod and were considered dead in the absence of any signs of movement. The larvae were considered, however, moribund if they only moved a little without showing any kind of swimming or vigorous movement. The moribund larvae could never revive and thus were counted as dead larvae. The extracts that failed to give $80 \%-100 \%$ mortality at $1000 \mathrm{ppm}$ after larval exposure for $24 \mathrm{~h}$ were rejected while extracts that resulted in mortality more than $80 \%$ after $24 \mathrm{~h}$ treatment were selected for further investigations.

\subsection{Larvicidal Bioassay with Selected Plant Extracts against} Ae. aegypti. In the second phase, the early fourth instars of Ae. aegypti were assayed with the selected extracts to assess the larvicidal efficacy of each extract in order to ascertain 
TABLE 2: Larvicidal potential of the leaf hexane extracts of the selected plant species against early fourth instars of dengue vector Aedes aegypti.

\begin{tabular}{lcccccccc}
\hline Name of the plant & $\mathrm{LC}_{50}(\mathrm{ppm})$ & $95 \%$ fiducial limits & $\mathrm{LC}_{90}(\mathrm{ppm})$ & 95\% fiducial limits & Regression coefficient & $\mathrm{SE}$ & $\chi^{2}$ & $\mathrm{DF}$ \\
\hline Achyranthes aspera & 82.555 & $73.554-91.860$ & 139.817 & $120.892-177.653$ & 5.600 & 0.851 & 6.738 & 6 \\
Cassia occidentalis & 117.451 & $104.353-138.047$ & 169.345 & $142.818-235.218$ & 8.064 & 1.515 & 1.569 & 3 \\
Catharanthus roseus & 86.913 & $75.720-102.674$ & 156.581 & $126.011-238.592$ & 5.012 & 0.92 & 5.978 & 4 \\
Lantana camara & 118.499 & $105.520-145.823$ & 159.985 & $134.032-242.868$ & 9.83 & 2.236 & 2.36 & 2 \\
Xanthium strumarium & 586.185 & $518.761-649.958$ & 851.821 & $754.752-1045.942$ & 7.895 & 1.403 & 0.561 & 3 \\
\hline
\end{tabular}

TABLE 3: Larvicidal potential of the stem hexane extracts of the selected plant species against early fourth instars of dengue vector Aedes aegypti.

\begin{tabular}{lcccccccc}
\hline Name of the plant & $\mathrm{LC}_{50}(\mathrm{ppm})$ & $95 \%$ fiducial limits & $\mathrm{LC}_{90}(\mathrm{ppm})$ & $95 \%$ fiducial limits & Regression coefficient & $\mathrm{SE}$ & $\chi^{2}$ & $\mathrm{DF}$ \\
\hline Achyranthes aspera & 68.133 & $42.340-92.723$ & 115.075 & $86.689-440.534$ & 5.630 & 0.901 & 15.910 & 5 \\
Cassia occidentalis & 149.698 & $135.101-166.024$ & 206.337 & $182.760-257.751$ & 9.195 & 1.724 & 0.433 & 3 \\
Catharanthus roseus & 108.245 & $82.320-196.106$ & 184.239 & $128.299-895.320$ & 5.548 & 0.980 & 7.609 & 4 \\
Lantana camara & 89.621 & $81.433-102.569$ & 125.892 & $108.091-180.782$ & 8.683 & 1.982 & 1.226 & 3 \\
Xanthium strumarium & 460.923 & $375.932-554.572$ & 1074.014 & $839.391-1638.379$ & 3.488 & 0.571 & 2.675 & 4 \\
\hline
\end{tabular}

their probable use in the fields. The bioassays were performed at $28 \pm 1^{\circ} \mathrm{C}$ in accordance with the procedure described by World Health Organization with slight modifications [29]. The graded series of each of the selected extracts was prepared using ethanol as the solvent. The bioassays were carried out at $20,40,60,80,100,200,400,600,800$, and $1000 \mathrm{ppm}$. Three replicates were carried out at once for each concentration of each extract. Controls were exposed to the solvent, that is, ethanol alone.

2.6. Data Analysis. The tests which caused more than $20 \%$ larval mortality in controls and resulted in more than $20 \%$ pupae formation in any bioassay were discarded and conducted again. In case the larval mortality in control bioassays ranged between $5 \%$ and $20 \%$, the control mortality was corrected using formula proposed by Abbott [30].

The obtained data was analysed by regression analysis using computerized statistical program SPSS (Version 22.0). The $\mathrm{LC}_{50}$ and $\mathrm{LC}_{90}$ values with $95 \%$ fiducial limits and chisquare were calculated in each bioassay for the assessment of significance and measurement of difference between the test samples.

\section{Results}

The screening for larvicidal potential of hexane and ethanol extracts prepared from the leaves and stems of five plant species, A. aspera, C. occidentalis, C. roseus L. camara, and $X$. strumarium, was carried out against early fourth instars of Ae. aegypti. The results obtained are presented in Table 1 which showed that $24 \mathrm{~h}$ exposure of the larvae with $1000 \mathrm{ppm}$ hexane extracts could result in $100 \%$ mortality, irrespective of the parts from which they were prepared. On the other hand, when the larvae of Ae. aegypti were exposed to the leaf and stem extracts prepared in ethanol, the larval mortality ranged between only $5.0 \%$ and $50.0 \%$ (Table 1 ). The least larval mortality of $5.0 \%$ was caused by the exposure to stem ethanol extract of L. camara and that of C. roseus, whereas leaf ethanol extract of $C$. roseus resulted in highest observed mortality of $50.0 \%$. Thus, all the ethanol extracts which could not result in significant larval mortality even at $1000 \mathrm{ppm}$ were rejected for further investigations.

When the larvicidal potentials of the leaf and stem hexane extracts of five plant species were evaluated against early fourth instars of Ae. aegypti, all the extracts revealed their potency to inflict mortality in early fourth instars of $A e$. aegypti (Tables 2 and 3). The data revealed that the larval exposure did not result in any pupal emergence indicating the selection of early instar stage. Moreover, the control or untreated group also did not exhibit any larval mortality or pupal emergence within $24 \mathrm{~h}$.

Our investigations clearly revealed the highest larvicidal potential of $A$. aspera extracts against Ae. aegypti early fourth instars, whether prepared from leaves or stems, exhibiting $\mathrm{LC}_{50}$ value of $82.555 \mathrm{ppm}$ and $68.133 \mathrm{ppm}$, respectively. The hexane leaf extract of $A$. aspera showed 5\% to $85.9 \%$ higher larvicidal activity in comparison to that shown by remaining hexane leaf extracts (Figure 4). On the other hand, when the larvae were exposed to the hexane extracts prepared from the stems, the $A$. aspera extract proved to be 0.23 - to 0.85 fold more efficient than the other four extracts (Figure 5). It is also significant to note the appreciable larvicidal efficacy of leaf hexane extract of $C$. roseus and stem hexane extract of $L$. camara revealing $\mathrm{LC}_{50}$ values of $86.913 \mathrm{ppm}$ and $89.621 \mathrm{ppm}$, respectively. Nevertheless, stem hexane extract of $C$. roseus and leaf hexane extract of L. camara could also result in noticeable larval mortality, the sudden rise in mortality noticed on exposure to $100 \mathrm{ppm}$ extracts (Figures 2 and 3).

On the other hand, $24 \mathrm{~h}$ exposure of the early fourth instars of Ae. aegypti to the leaf and stem hexane extracts of $X$. strumarium resulted in moderate toxic effects, the respective $\mathrm{LC}_{50}$ values obtained being $586.185 \mathrm{ppm}$ and $460.923 \mathrm{ppm}$ (Tables 2 and 3; Figures 4 and 5). The results showed that exposure to $100 \mathrm{ppm}$ of leaf and stem hexane extracts of $X$. strumarium could not cause any larval mortality (Figures 2 and 3) whereas, on increasing the exposure concentration to 


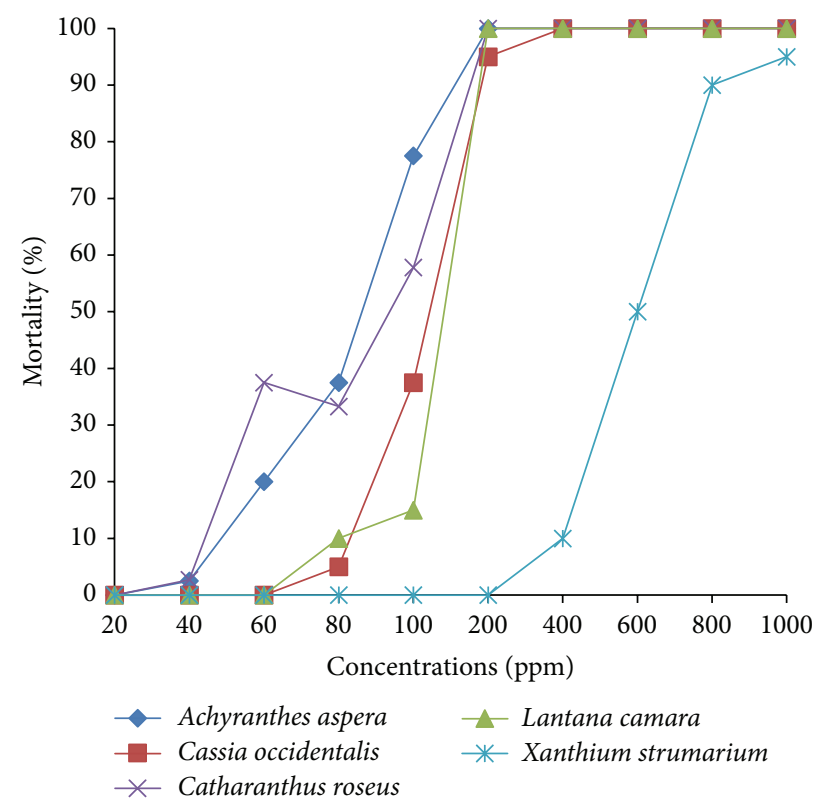

Figure 2: Percent mortality of hexane leaf extracts of different selected plants at different concentrations against early IV instars of Ae. aegypti.

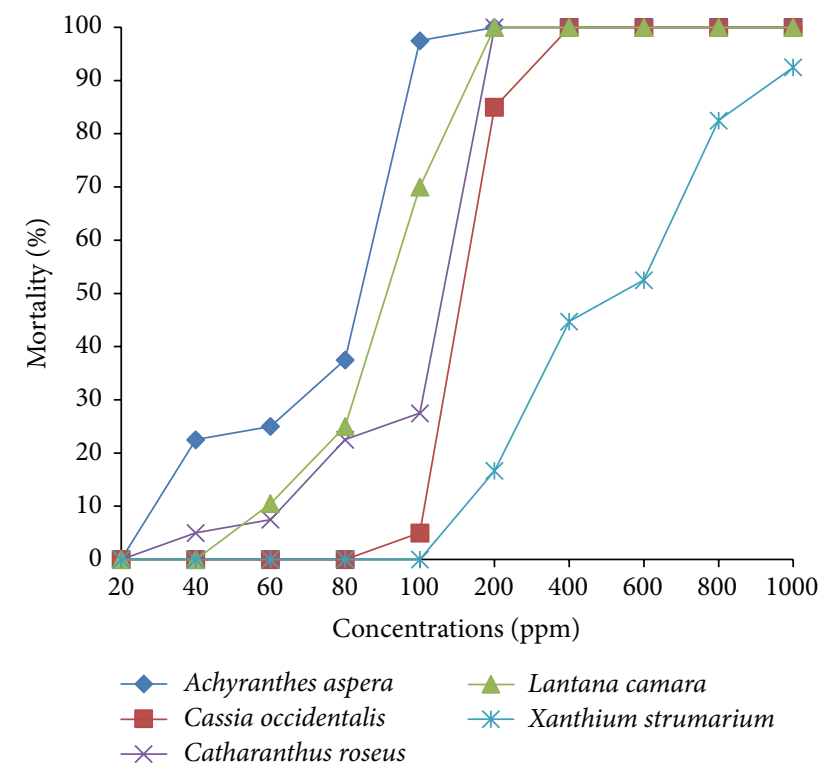

FIGURE 3: Percent mortality of hexane stem extracts of different selected plants at different concentrations against early IV instars of Ae. aegypti.

$200 \mathrm{ppm}$, the hexane leaf extract was found to be ineffective, while the hexane stem extract could result in only $17 \%$ larval mortality as compared to $100 \%$ mortality obtained on exposure to $A$. aspera extracts.

On the contrary, when the early fourth instars of $A e$. aegypti were exposed to $C$. occidentalis extracts, the hexane leaf extract showed higher efficacy as compared to the hexane stem extract, the respective $\mathrm{LC}_{50}$ values being $117.451 \mathrm{ppm}$ and $149.698 \mathrm{ppm}$ (Tables 2 and 3; Figures 4 and 5). It is

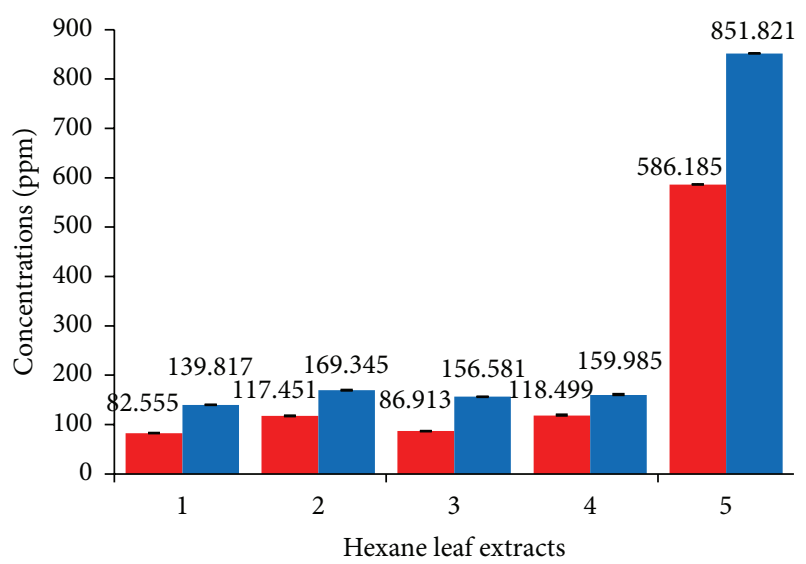
(1) Achyranthes aspera
- $\mathrm{LC}_{50}$
(2) Cassia occidentalis
- $\mathrm{LC}_{90}$
(3) Catharanthus roseus
(4) Lantana camara
(5) Xanthium strumarium

FIgURE 4: Comparative larvicidal activity of hexane leaf extract of different selected plants against early IV instars of Ae. aegypti.

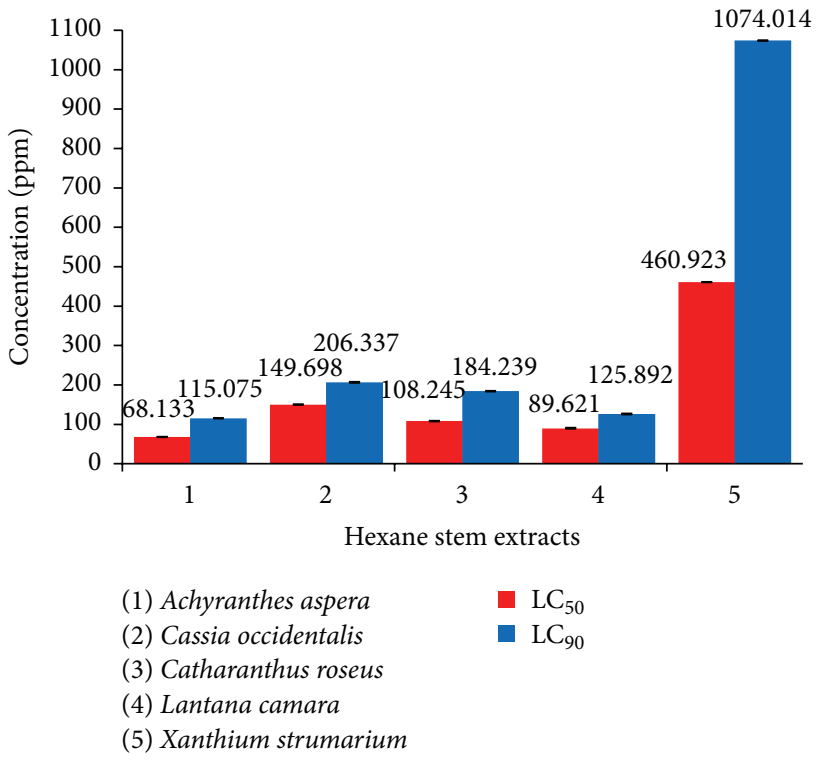

FIgURE 5: Comparative larvicidal activity of hexane stem extracts of different selected plants against early IV instars of Ae. aegypti.

remarkable to note that both the leaf hexane extract and leaf stem extract did not cause any appreciable mortality at lower concentrations after which a steep rise in larval mortality was noticed (Figures 2 and 3 ).

\section{Discussion}

The extensive use of synthetic chemical insecticides results in environmental degradation, hazards, and resistance in major vector species and this has necessitated leading the way towards the development of a more potent and environmentally friendly insecticide. Nowadays, the control of 
mosquitoes at larval stage is focused on the use of plant extracts. Plants produce various chemicals, many of which have medicinal, insecticidal, repellent, and growth regulatory properties [11].

The present investigations clearly revealed that when 1000 ppm hexane and ethanol stem and leaf extracts were screened for their larvicidal efficacy against early IV instars of Ae. aegypti, the hexane extracts exhibited significant larvicidal efficacy causing $100 \%$ larval mortality. Similar results were reported by Kumar et al. [5] who performed an initial screening of fifteen local plant species to explore their potential as a mosquito larvicidal agent against early fourth instars of dengue vector Ae. aegypti and reported the effectual larvicidal potential of hexane extracts of selected plant species resulting in $100 \%$ mortality at $1000 \mathrm{ppm}$. They observed that the extracts made from different parts of A. aspera, C. occidentalis, L. camara, Ricinus communis, Trachyspermum ammi, and Zingiber officinalis possessed significant larvicidal potential with $\mathrm{LC}_{50}$ values ranging from $30.00 \mathrm{ppm}$ to $74.67 \mathrm{ppm}$. Likewise, Kumar et al. [16] when assaying different parts of a weed, Parthenium hysterophorus, extracted in different solvents against Ae. aegypti larvae found only hexane and petroleum ether extracts effective causing $100 \%$ mortality.

Likewise, evaluation of the larvicidal activity of extracts prepared from fourteen medicinal plants against Ae. aegypti revealed that eight out of fourteen plant species resulted in $100 \%$ larval mortality with their $\mathrm{LC}_{50}$ values ranging between $4.1 \mu \mathrm{g} / \mathrm{mL}$ and $89.4 \mu \mathrm{g} / \mathrm{mL}$ [31]. Similar studies performed by Sakthivadivel and Daniel [32] evidently proved the toxicity of six plant extracts, Acacia nilotica (leaf), A. mexicana (leaves and seeds), Citrullus colocynthis (leaf), Jatropha curcas (leaf), and Withania somnifera (leaf), resulting in an $\mathrm{LC}_{50}$ value of less than 100 ppm against 3 rd instars of $C x$. quinquefasciatus, An. stephensi, and Ae. aegypti. Comparable screening assays with a total of 94 extracts prepared from ten plant species belonging to eight families widely found in the Northeast of Brazil were performed by Oliveira et al. [33]. The assays were carried out with $250 \mu \mathrm{g} / \mathrm{mL}$ extracts against fourth instars of Ae. aegypti which showed significant toxicity (>75\%) of nineteen extracts from six plant species, Coccoloba mollis, Guettarda grazielae, Merremia aegyptia, Rourea doniana, Spermacoce verticillata, and Triplaris americana. Contrary results were presented by Yang et al. [34] who studied the cidal effects of polar methanol extracts, prepared from 28 medicinal plant species, against early 4 th instars of Ae. aegypti and observed $100 \%$ larval mortality at $100 \mathrm{ppm}$ methanol extracts of Kaempferia galanga rhizome.

Our results confirmed greater larvicidal potential of nonpolar hexane extracts of all the five plant species against Ae. aegypti than the polar ethanol extracts which led us to assess their effectiveness at various concentrations. Our studies are in conformity with that of Rahuman et al. [35] who obtained better potency of nonpolar extracts than the polar extracts when they assayed the ethyl acetate, butanol, and petroleum ether extracts of five species of Euphorbiaceae family, Jatropha curcas, Pedilanthus tithymaloides, Phyllanthus amarus, Euphorbia hirta, and E. tirucalli against the early fourth instars of Ae. aegypti.
In contrast, Kalimuthu et al. [36] established the highest larval mortality in Ae. aegypti when exposed to the ethanol leaf extract of Cadaba indica lam exhibiting an $\mathrm{LC}_{50}$ value of $143.75 \mathrm{ppm}$ compared with that resulting on exposure to hexane, chloroform, and petroleum ether extracts. However, results comparable to present investigations were reported by Maheswaran et al. [37] who tested the crude extracts of Leucas aspera for their larvicidal activity against Ae. aegypti and $C x$. quinquefasciatus and observed hexane extract to possess the highest larvicidal activity against the two vectors followed by that of chloroform and ethanol. Likewise, the studies reported by Warikoo and Kumar [17] showed hexane and petroleum ether extracts of Argemone mexicana as effective larvicides resulting in $80-100 \%$ mortality at $1,000 \mathrm{ppm}$ when assayed against fourth instars of Ae. aegypti. Other extracts, benzene, acetone, and ethanol, however, were found to be comparatively ineffective.

Present investigations clearly revealed the efficacy of hexane extracts prepared from the leaves and stem of $A$. aspera against early IV instars of Ae. aegypti as compared to the other four extracts. Similar results have been reported by Bhattacharya and Chandra [38] who assayed different instars of $C x$. vishnui with acetone leaf extracts of $A$. aspera and reported $\mathrm{LC}_{50}$ values ranging from $35.46 \mathrm{ppm}$ to $63.39 \mathrm{ppm}$. Earlier reports have shown the larvicidal efficacy of the extracts prepared from the leaves, flowers, and the seeds of A. aspera against the fourth instars of An. subpictus and $C x$. tritaeniorhynchus [19]. In contrast, Kumar et al. [5] found the higher larvicidal efficacy of hexane leaf extract of $L$. camara and $C$. occidentalis exhibiting a significant $\mathrm{LC}_{50}$ value of $30.71 \mathrm{ppm}$ and $74.67 \mathrm{ppm}$ as compared to that of $A$. aspera. Rajasekaran and Duraikannan [39] also reported higher toxicity of petroleum ether and aqueous extract of L. camara against 4th instars of Ae. aegypti resulting in 100\% mortality as compared to the chloroform extract which could result in only $36.89 \%$ mortality after 24 hours. The petroleum ether and $n$-butanol extracts of $C$. occidentalis are also found to be more selective and biodegradable agents with appreciable larvicidal potential against filarial vector $C x$. quinquefasciatus [23].

\section{Conclusions}

Our investigations have established the potential of hexane leaf extracts prepared from different plant species against early fourth instars of Ae. aegypti and their possible use in the development of larvicides for mosquito management. The variety of types and levels of active constituents in each kind of extract may be responsible for the variability in their potential against Ae. aegypti. However, the mechanism involved causing mortality of mosquito larvae is still unknown and needs to be studied further. Our investigations need further exploration to find out and identify the bioactive components involved and their mode of action.

\section{Conflict of Interests}

The authors declare that they have no conflict of interests regarding the publication of this paper. 


\section{Acknowledgment}

The authors are highly thankful to Dr. Savithri Singh, Principal, Acharya Narendra Dev College, University of Delhi, for providing infrastructure and research facilities.

\section{References}

[1] World Health Organization (WHO), "Dengue and severe dengue," 2015, http://www.who.int/mediacentre/factsheets/fs117/en/.

[2] National Vector Borne Disease Control Programme (NVBDCP), "Dengue Cases and Deaths in the Country," 2015, http://nvbdcp .gov.in/dengue6.html.

[3] N. Liu, Q. Xu, F. Zhu, and L. Zhang, "Pyrethroid resistance in mosquitoes," Insect Science, vol. 13, no. 3, pp. 159-166, 2006.

[4] K. Raghvendra, "Chemical insecticides in malaria vector control in India," ICMR Bulletin, vol. 32, no. 10, pp. 1-7, 2002.

[5] S. Kumar, N. Wahab, M. Mishra, and R. Warikoo, "Evaluation of 15 local plant species as larvicidal agents against an Indian strain of dengue fever mosquito, Aedes aegypti L. (Diptera: Culicidae)," Frontiers in Physiology, vol. 3, article 104, pp. 1-6, 2012.

[6] S. N. Tikar, M. J. Mendki, K. Chandel, B. D. Parashar, and S. Prakash, "Susceptibility of immature stages of Aedes (Stegomyia) aegypti; vector of dengue and chikungunya to insecticides from India," Parasitology Research, vol. 102, no. 5, pp. 907913, 2008.

[7] H. P. Borase, C. D. Patil, R. B. Salunkhe, C. P. Narkhede, B. K. Salunke, and S. V. Patil, "Phyto-synthesized silver nanoparticles: a potent mosquito biolarvicidal agent," Journal of Nanomedicine and Biotherapeutic Discovery, vol. 3, article 111, 2013.

[8] M. B. Isman, "Plant essential oils for pest and disease management," Crop Protection, vol. 19, no. 8-10, pp. 603-608, 2000.

[9] D. Rajmohan, S. Anbarasi, and K. L. Kumar, "Efficacy of Vinca rosea (Apocynaneae) against the growth and development of the chikungunya vector, Aedes aegypti L. (Diptera: Culucidae)," International Journal of Pharmacy and Pharmaceutical Sciences, vol. 4, no. 4, pp. 50-51, 2012.

[10] K. Sukumar, M. J. Perich, and L. R. Boom-Bar, "Botanical derivatives in mosquito control: a review," Journal of the American Mosquito Control Association, vol. 7, no. 2, pp. 210-237, 1991.

[11] A. Ghosh, N. Chowdhury, and G. Chandra, "Plant extracts as potential mosquito larvicides," Indian Journal of Medical Research, vol. 135, no. 5, pp. 581-598, 2012.

[12] A. Amer and H. Mehlhorn, "Larvicidal effects of various essential oils against Aedes, Anopheles and Culex larvae (Diptera, Culicidae)," Parasitology Research, vol. 99, no. 4, pp. 466-472, 2006.

[13] A. Amer and H. Mehlhorn, "Repellency effect of forty-one essential oils against Aedes, Anopheles, and Culex mosquitoes," Parasitology Research, vol. 99, no. 4, pp. 478-490, 2006.

[14] A. A. Rahuman, A. Bagavan, C. Kamaraj et al., "Evaluation of indigenous plant extracts against larvae of Culex quinquefasciatus Say (Diptera: Culicidae)," Parasitology Research, vol. 104, no. 3, pp. 637-643, 2009.

[15] A. A. Rahuman, A. Bagavan, C. Kamaraj, E. Saravanan, A. A. Zahir, and G. Elango, "Efficacy of larvicidal botanical extracts against Culex quinquefasciatus Say (Diptera: Culicidae)," Parasitology Research, vol. 104, no. 6, pp. 1365-1372, 2009.

[16] S. Kumar, G. Nair, A. P. Singh, S. Batra, N. Wahab, and R. Warikoo, "Evaluation of the larvicidal efficiency of stem, roots and leaves of the weed, Parthenium hysterophorus (Family:
Asteraceae) against Aedes aegypti L.", Asian Pacific Journal of Tropical Disease, vol. 2, no. 5, pp. 395-400, 2012.

[17] R. Warikoo and S. Kumar, "Impact of Argemone mexicana extracts on the cidal, morphological, and behavioral response of dengue vector, Aedes aegypti L. (Diptera: Culicidae)," Parasitology Research, vol. 112, no. 10, pp. 3477-3484, 2013.

[18] R. Warikoo and S. Kumar, "Oviposition altering and ovicidal efficacy of root extracts of Argemone mexicana against dengue vector, Aedes aegypti (Diptera: Culicidae)," Journal of Entomology and Zoology Studies, vol. 2, no. 4, pp. 11-17, 2014.

[19] A. Bagavan, A. A. Rahuman, C. Kamaraj, and K. Geetha, "Larvicidal activity of saponin from Achyranthes aspera against Aedes aegypti and Culex quinquefasciatus (Diptera: Culicidae)," Parasitology Research, vol. 103, no. 1, pp. 223-229, 2008.

[20] A. Dey, "Achyranthes aspera L.: phytochemical and pharmacological aspects," International Journal of Pharmaceutical Sciences Review and Research, vol. 9, no. 2, pp. 72-82, 2011.

[21] V. M. Vashishtha, T. J. John, and A. Kumar, "Clinical and pathological features of acute toxicity due to Cassia occidentalis in vertebrates," Indian Journal of Medical Research, vol. 130, no. 1, pp. 23-30, 2009.

[22] J. P. Yadav, V. Arya, S. Yadav, M. Panghal, S. Kumar, and S. Dhankhar, "Cassia occidentalis L.: a review on its ethnobotany, phytochemical and pharmacological profile," Fitoterapia, vol. 81, no. 4, pp. 223-230, 2010.

[23] D. Kumar, R. Chawla, P. Dhamodaram, and N. Balakrishnan, "Larvicidal activity of Cassia occidentalis (Linn.) against the larvae of bancroftian filariasis vector mosquito Culex quinquefasciatus," Journal of Parasitology Research, vol. 2014, Article ID 236838, 5 pages, 2014.

[24] S. Subarani, S. Sabhanayakam, C. Kamaraj, G. Elango, and M. A. Kadir, "Efficacy of larvicidal and pupicidal activity of Catharanthus roseus aqueous and solvent extracts against Anopheles stephensi Liston and Culex quinquefasciatus Say (Diptera: Culicidae)," Asian Pacific Journal of Tropical Medicine, vol. 6, no. 8, pp. 625-630, 2013.

[25] J. M. Joy, S. Vamsi, C. Satish, and K. Nagaveni, "Lantana camara Linn: a review," International Journal of Phytotherapy, vol. 2, no. 2, pp. 66-73, 2012.

[26] N. M. Reddy, "Lantana Camara Linn. Chemical constituents and medicinal properties: a review," Scholars Academic Journal of Pharmacy, vol. 2, no. 6, pp. 445-448, 2013.

[27] S. P. Singh, K. Raghavendra, and V. K. Dua, "Evaluation of larvicidal and repellent activity of Xanthium strumarium (Asteraceae) ethanol extract against mosquito vectors," Journal of Communicable Diseases, vol. 41, no. 4, pp. 263-269, 2009.

[28] S. Kumar, R. Warikoo, and N. Wahab, "Larvicidal potential of ethanolic extracts of dried fruits of three species of peppercorns against different instars of an Indian strain of dengue fever mosquito, Aedes aegypti L. (Diptera: Culicidae)," Parasitology Research, vol. 107, no. 4, pp. 901-907, 2010.

[29] WHO Report, World Malaria Report, WHO, UNICEF, Geneva, Switzerland, 2005.

[30] W. S. Abbott, "A method of computing the effectiveness of an insecticide," Journal of Economic Entomology, vol. 18, no. 2, pp. 265-267, 1925.

[31] S. Promsiri, A. Naksathi, M. Kruatrachue, and U. Thavara, "Evaluation of larvicidal activity of medicinal plant extracts to Aedes aegypti (Diptera: Culicidae) and other effects on a nontarget fish," Journal of American Mosquito Control Association, vol. 22, pp. 292-295, 2006. 
[32] M. Sakthivadivel and T. Daniel, "Evaluation of certain insecticidal plants for the control of vector mosquitoes viz. Culex quinquefasciatus, Anopheles stephensi and Aedes aegypti," Applied Entomology and Zoology, vol. 43, no. 1, pp. 57-63, 2008.

[33] P. V. Oliveira, J. C. Ferreira Jr., F. S. Moura et al., "Larvicidal activity of 94 extracts from ten plant species of northeastern of Brazil against Aedes aegypti L. (Diptera: Culicidae)," Parasitology Research, vol. 107, no. 2, pp. 403-407, 2010.

[34] Y.-C. Yang, I.-K. Park, E.-H. Kim, H.-S. Lee, and Y.-J. Ahn, "Larvicidal activity of medicinal plant extracts against Aedes aegypti, Ochlerotatus togoi, and Culex pipiens pallens (Diptera: Culicidae)," Journal of Asia-Pacific Entomology, vol. 7, no. 2, pp. 227-232, 2004.

[35] A. A. Rahuman, G. Gopalakrishnan, P. Venkatesan, and K. Geetha, "Larvicidal activity of some Euphorbiaceae plant extracts against Aedes aegypti and Culex quinquefasciatus (Diptera: Culicidae)," Parasitology Research, vol. 102, no. 5, pp. 867-873, 2008.

[36] K. Kalimuthu, K. Murugan, C. Panneerselvam, and J. S. Hwang, "Mosquito larvicidal activity of Cadaba indica lam leaf extracts against the dengue vector, Aedes aegypti," Asian Journal of Plant Science and Research, vol. 2, no. 5, pp. 633-637, 2012.

[37] R. Maheswaran, S. Sathish, and S. Ignacimuthu, "Larvicidal activity of Leucas aspera (Wild.) against the larvae of Culex quinquefasciatus say and Aedes aegypti L.," International Journal of Integrated Biology, vol. 2, no. 3, pp. 214-217, 2008.

[38] K. Bhattacharya and G. Chandra, "Bioactivity of Acyranthes aspera (Amaranthaceae) foliage against the Japanese encephalitis vector Culex vishnui group," Journal of Mosquito Research, vol. 3, no. 13, pp. 89-96, 2013.

[39] A. Rajasekaran and G. Duraikannan, "Larvicidal activity of plant extracts on Aedes aegypti L," Asian Pacific Journal of Tropical Biomedicine, vol. 2, no. 3, pp. S1578-S1582, 2012. 

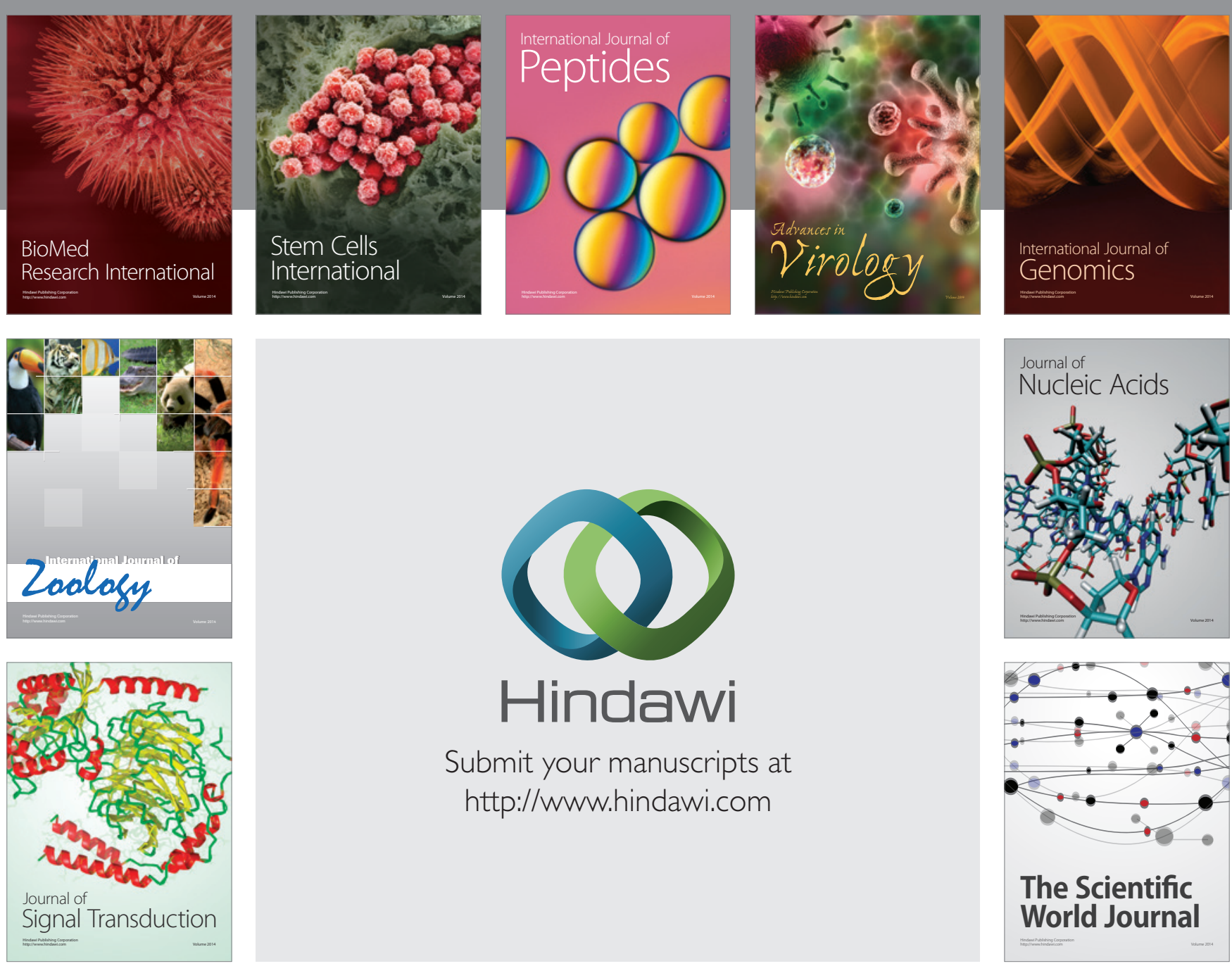

Submit your manuscripts at

http://www.hindawi.com
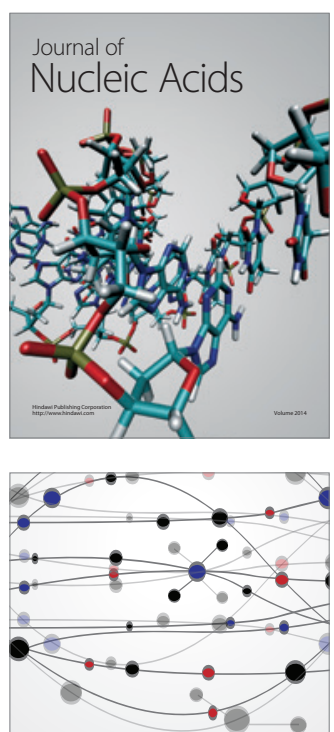

The Scientific World Journal
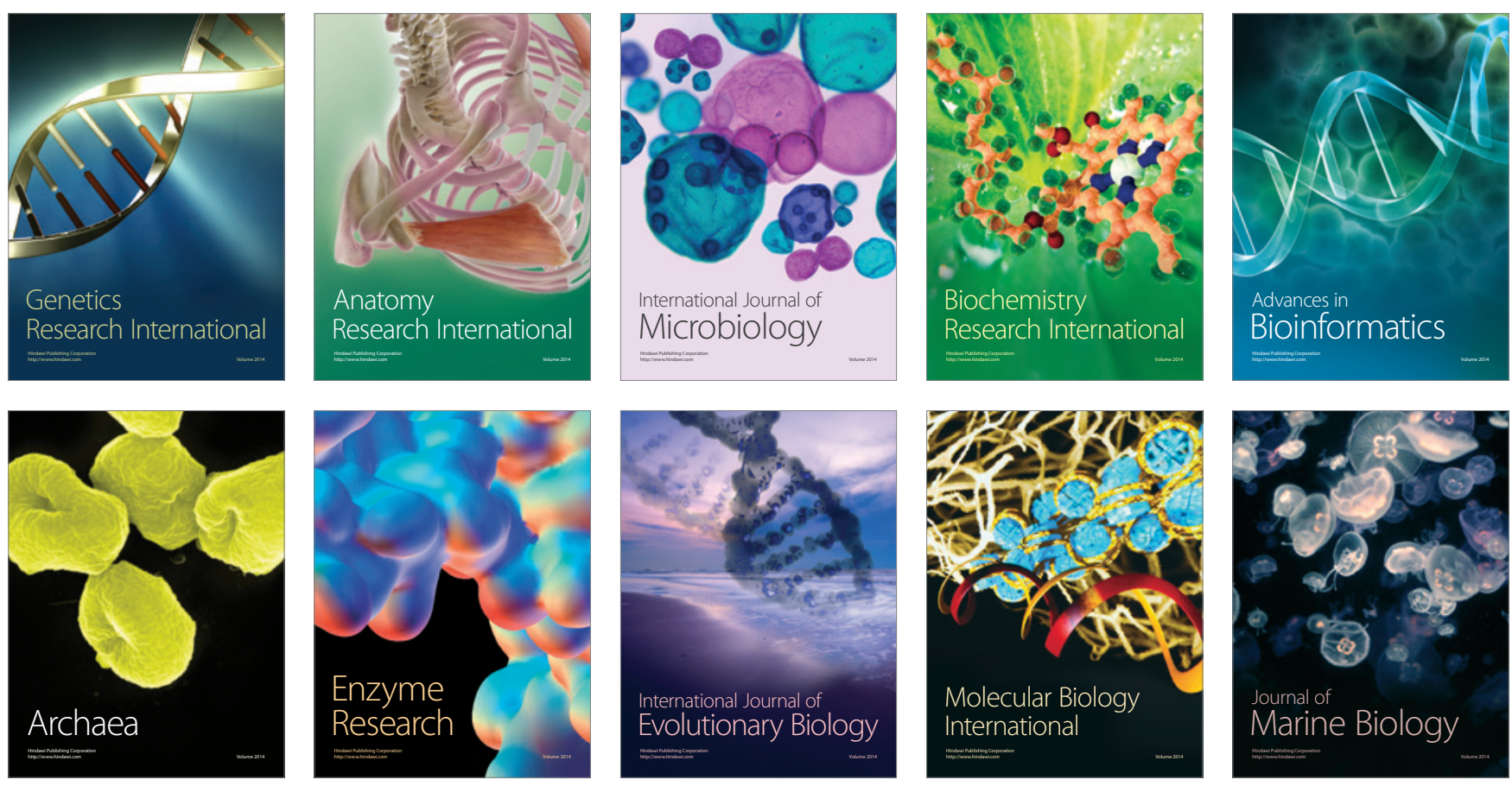